\title{
Knowledge and Social Laws
}

\author{
Wiebe van der Hoek Mark Roberts Michael Wooldridge \\ Department of Computer Science \\ University of Liverpool \\ Liverpool L69 7ZF, UK \\ \{wiebe,mark, mjw\}@csc.liv.ac.uk
}

\begin{abstract}
In this paper we combine existing work in the area of social laws with a framework for reasoning about knowledge in multi-agent systems. The unifying framework in which this is done is based on Alternating-time Temporal Logic (ATL), to which semantics we add epistemic accessibility relations (to deal with the knowledge), actions (in order to naturally talk about allowed and forbidden actions) and updates (to model the effect of the implementation of the constraint in a social law). Apart from a constraint, a social law has an objective: in our formalism, such objectives may refer to the knowledge that agents possess or do not possess. The result is a framework in which we can, for example, express that a desirable property (objective) of a social law is that one agent has the ability to bring about a certain type of knowledge in another agent, or that if one agent knows something, then it should behave in a certain way. We illustrate our approach with a case study, and we use model checking to demonstrate that properties of social laws with respect to this case study.
\end{abstract}

\section{Categories and Subject Descriptors}

I.2.4 [Artificial Intelligence]: Knowledge Representation Formalisms and Methods-Representation languages, Modal logic, Temporal logic; I.2.11 [Artificial Intelligence]: Distributed Artificial Intelligence - Coherence and coordination, Multiagent systems

\section{General Terms}

Theory, Design, Verification

\section{Keywords}

Social Laws, Alternating-time Temporal Epistemic Logic, Knowledge

Permission to make digital or hard copies of all or part of this work for personal or classroom use is granted without fee provided that copies are not made or distributed for profit or commercial advantage and that copies bear this notice and the full citation on the first page. To copy otherwise, to republish, to post on servers or to redistribute to lists, requires prior specific permission and/or a fee.

AAMAS'05, July 25-29, 2005, Utrecht, Netherlands.

Copyright 2005 ACM 1-59593-094-9/05/0007 ...\$5.00.

\section{INTRODUCTION}

A social law is a restriction on the behaviour of agents, to ensure they can work individually in a mutually compatible manner in order to fulfil their individual goals, and possibly joint goals. Social laws work by prohibiting the performance of certain actions in certain situations (states). The social laws paradigm was first proposed by Moses, Shoham and Tennenholtz $[11,10,12,8,13,14]$.

In [16], van der Hoek et al extend the social laws framework further. They show that Alternating-time Temporal Logic (ATL) provides a rich and natural technical framework within which to investigate social laws and their properties. In this framework, a social law consists of two parts: an objective of the law, written as an ATL specification, and a behavioural constraint, which is a function that for every action returns the set of states where that action is forbidden from being performed. The objective of the law represents what the society aims to achieve by adopting the law, and the behavioural constraint corresponds to the requirements that the law places on the members of society.

With social laws expressed in this way, the problem of determining whether a social law is effective corresponds to an ATL model checking problem. This is advantageous as it allows the use of existing ATL model checkers. Expressing social laws in ATL in this way clearly has other advantages to it. Social laws frequently refer to the powers or rights (or, conversely, the limits to powers) that agents have, so ATL, with its cooperation modalities, is a very natural choice to express such laws. Also, ATL has the ability to express laws that refer to conditions that must hold over time. Finally, [16] studies various computational problems in their new framework of social laws, and found the complexity of the feasibility problem for social laws in the most general case to be no more complex than the corresponding problem in the Shoham-Tennenholtz framework (it is NP-complete).

However, the framework of [16] is based on quite a strong and unrealistic assumption that agents know everything about the state of the system. This is modelled with a global state space which all agents have access to. In this paper we do away with the need for this assumption by extending the framework further by incorporating the notion of knowledge. Knowledge is important in any non-trivial multi-agent system, and as knowledge will come to play in almost any coordination scenario, we can clearly see a need to extend our framework. Not only will the agents' knowledge of the system be used in order to follow certain laws, but we can have laws in which certain information must become known (or remain private) to an agent or a coalition of agents. 
So, by imposing certain social laws, we can force desirable knowledge properties to hold.

Furthermore, we can have laws which are only known to a certain agent or group of agents, and only these agents need follow the law. In this paper, we demonstrate how to 'transform' the objective of a social law in several, individual objectives, which have a feasible format: depending on the information the agent has, he is required to achieve some situation that is under his control.

This paper is structured as follows: We begin by introducing Action-based Alternating Epistemic Transition Systems (AAETSS), based on an essentially equivalent version of the structures of [16] but extended in order to represent the knowledge of the agents; we then introduce Alternating Temporal Epistemic Logic (ATEL) itself, the logic we will use to express social laws. In section 4 we formally define this new extended framework of social laws with respect to AAETS and ATEL; and then in section 5 we present a case study to illustrate the power of our framework and to show how model checking can be performed using standard ATL model checkers. A discussion of related work is presented in section 6 , while section 7 concludes.

\section{SEMANTIC STRUCTURES}

In [16] the semantic structures used are known as Actionbased Alternating Transition Systems (AATSs) and are essentially equivalent to the alternating transition systems used by Alur and colleagues to give semantics to ATL (see [2]). In this section we extend AATSs into what we call Action-based Alternating Epistemic Transition Systems (AAETSs). The main difference is that we introduce an epistemic accessibility relation for each agent in the system, which is used to capture each agent's knowledge. The approach is standard in the literature of epistemic logic $[5,7]$.

We first assume that the systems of interest to us may be in any of a finite set $Q$ of possible states, with some $q_{0} \in Q$ designated as the initial state. Systems are populated by a set $\mathrm{Ag}$ of agents; a coalition of agents is simply a set $G \subseteq A g$, and the set of all agents is known as the grand coalition. Each agent $i \in A g$ is associated with a set $A c_{i}$ of possible actions, and we assume that these sets of actions are pairwise disjoint. We denote the set of actions associated with a coalition $G \subseteq A g$ by $A c_{G}$, so $A c_{G}=\bigcup_{i \in G} A c_{i}$. A joint action for a coalition $G$ is an indexed tuple $\left\langle\alpha_{1}, \ldots, \alpha_{k}\right\rangle$, where $\alpha_{i} \in A c_{i}$, for each $i \in G$. We denote the set of all joint actions for coalition $G$ by $J_{G}$, so $J_{G}=\prod_{i \in G} A c_{i}$. Given an element $j$ of $J_{G}$ and agent $i \in G$, we denote $i$ 's component of $j$ by $j_{i}$. Now, for each agent $i \in A g$, we also have an epistemic accessibility relation $\sim_{i}$, which represents indistinguishable states to agent $i$, used to capture $i$ 's knowledge. Thus, an AAETs is an $(2 n+7)$-tuple

$$
\left\langle Q, q_{0}, A g, A c_{1}, \ldots, A c_{n}, \sim_{1}, \ldots, \sim_{n}, \rho, \tau, \Phi, \pi\right\rangle
$$

where:

- $Q$ is a finite, non-empty set of states;

- $q_{0} \in Q$ is the initial state;

- $A g=\{1, \ldots, n\}$ is a finite, non-empty set of agents;
- $A c_{i}$ is a finite, non-empty set of actions, for each $i \in$ $A g$, where $A c_{i} \cap A c_{j}=\emptyset$ for all $i \neq j \in A g$;

- $\sim{ }_{i} \subseteq Q \times Q$ is an epistemic accessibility relation for each agent $i \in A g$. Each $\sim_{i}$ must be an equivalence relation;

- $\rho: A c_{A g} \rightarrow 2^{Q}$ is an action precondition function, which for each action $\alpha \in A c_{A g}$ defines the set of states $\rho(\alpha)$ from which $\alpha$ may be executed;

- $\tau: Q \times J_{A g} \rightarrow Q$ is a partial system transition function, which defines the state $\tau(q, j)$ that would result by the performance of $j$ from state $q$;

- $\Phi$ is a finite, non-empty set of atomic propositions; and

- $\pi: Q \rightarrow 2^{\Phi}$ is an interpretation function, which gives the set of primitive propositions satisfied in each state: if $p \in \pi(q)$, then proposition $p$ is true in state $q$.

AAETSs must satisfy two coherence constraints:

1. Non-triviality [8].

Agents always have at least one legal action: $\forall q \in$ $Q, \forall i \in A g, \exists \alpha \in A c_{i}$ s.t. $q \in \rho(\alpha)$

\section{Consistency.}

The $\rho$ and $\tau$ functions agree on actions that may be performed: $\forall q, \forall j \in J_{A g},(q, j) \in \operatorname{dom} \tau$ iff $\forall i \in A g, q \in$ $\rho\left(j_{i}\right)$

We denote the set of sequences over $Q$ by $Q^{*}$, and the set of non-empty sequences over $Q$ by $Q^{+}$.

For a coalition of agents $G \subseteq A g$, we denote the union of $G$ 's accessibility relations by $\sim_{G}^{E}$, so $\sim_{G}^{E}=\left(\bigcup_{i \in G} \sim_{i}\right)$. The transitive closure of $\sim_{G}^{E}$ is denoted by $\sim_{G}^{C}$.

Given an agent $i \in A g$ and a state $q \in Q$, we denote the options available to $i$ in $q$ by options $(i, q)=\{\alpha \mid \alpha \in$ $A c_{i}$ and $\left.q \in \rho(\alpha)\right\}$. We then say that a strategy for an agent $i \in A g$ is a function: $\sigma_{i}: Q \rightarrow A c_{i}$ which must satisfy the legality constraint that $\sigma_{i}(q) \in \operatorname{options}(i, q)$ for all $q \in Q$.

A strategy profile for a coalition $G=\left\{a_{1}, \ldots, a_{k}\right\} \subseteq A g$ is a tuple of strategies $\left\langle\sigma_{1}, \ldots, \sigma_{k}\right\rangle$, one for each agent $a_{i} \in$ $G$. We denote by $\Sigma_{G}$ the set of all strategy profiles for coalition $G \subseteq A g$; if $\sigma_{G} \in \Sigma_{G}$ and $i \in G$, then we denote $i$ 's component of $\sigma_{G}$ by $\sigma_{G}^{i}$. Given a strategy profile $\sigma_{G} \in \Sigma_{G}$ and state $q \in Q$, let $\operatorname{out}\left(\sigma_{G}, q\right)$ denote the set of possible states that may result by the members of the coalition $G$ acting as defined by their components of $\sigma_{G}$ for one step from $q$. Formally, out $\left(\sigma_{G}, q\right)=$

$$
\left\{q^{\prime} \mid \tau(q, j)=q^{\prime} \text { where } \sigma_{G}^{i}(q)=j_{i} \text { for } i \in G\right\}
$$

Notice that, for any grand coalition strategy profile $\sigma_{A g}$ and state $q$, the set out $\left(\sigma_{A g}, q\right)$ will be singleton.

A computation $\lambda$ is an infinite sequence of states $\lambda=$ $q_{0}, q_{1}, \ldots$ A computation $\lambda \in Q^{+}$starting in state $q$ is referred to as a $q$-computation; if $u \in \mathbb{N}$, then we denote by 
$\lambda[u]$ the component indexed by $u$ in $\lambda$ (thus $\lambda[0]$ denotes the first element, $\lambda[1]$ the second, and so on).

Given a strategy profile $\sigma_{G}$ for some coalition $G$, and a state $q \in Q$, we define $\operatorname{comp}\left(\sigma_{G}, q\right)$ to be the set of possible runs that may occur if every agent $a_{i} \in G$ follows the corresponding strategy $\sigma_{i}$, starting when the system is in state $q \in Q$. Formally, $\operatorname{comp}\left(\sigma_{G}, q\right)=$

$$
\left\{\lambda \mid \lambda[0]=q \text { and } \forall u \in \mathbb{N}: \lambda[u+1] \in \operatorname{out}\left(\sigma_{G}, \lambda[u]\right)\right\} .
$$

\section{ATEL}

In this section we define the language used to express social laws in our framework. Alternating temporal epistemic logic (ATEL) [18] is an extension of the well known ATL of Alur, Henzinger, and Kupferman, which in turn can be understood as a generalisation of the branching time temporal logic CTL. As with ATL, we have cooperation modalities, such as, $\langle\langle G\rangle\rangle \varphi$, which expresses that the coalition $G$ can cooperate to ensure $\varphi$; more precisely, that there exists a strategy profile for $G$ such that by following this strategy profile, $G$ can ensure $\varphi$.

The " $\square$ " temporal operator means "now and forever more": additional temporal connectives in ATEL are " $\nabla$ " ("either now or at some point in the future"), " $\mathcal{U}$ " ("until"), and " $\bigcirc$ " ("in the next state"). Additionally, we have the following knowledge operators: $K_{i} \varphi$ ("agent $i$ knows $\varphi$ "), $E_{G} \varphi$ ("everyone in coalition $G$ knows $\varphi$ "), and $C_{G} \varphi$ ("it is common knowledge to everyone in the coalition $G$ that $\varphi$ ").

Formally, the set of ATEL formulae, formed with respect to a set of agents $A g$, and a set of primitive propositions $\Phi$, is given by the following grammar:

$$
\begin{aligned}
\varphi \quad:= & p|\neg \varphi| \varphi \vee \varphi\left|K_{i} \varphi\right| E_{G} \varphi\left|C_{G} \varphi\right| \\
& \langle\langle G\rangle \bigcirc \varphi|\langle\langle G\rangle \square \varphi|\langle\langle G\rangle \varphi \mathcal{U} \varphi
\end{aligned}
$$

where $p \in \Phi$ is a propositional variable, $G \subseteq A g$ is a set of agents, and $i \in A g$ is an agent.

We now give the truth definition of ATEL formulae on an AAETS $S$ and a state $q$ :

$$
\begin{aligned}
& S, q \models p \text { iff } p \in \pi(q) \quad \text { (where } p \in \Phi) ; \\
& S, q \models \neg \varphi \text { iff } S, q \not \models \varphi ; \\
& S, q \models \varphi \vee \psi \text { iff } S, q \models \varphi \text { or } S, q \models \psi ; \\
& S, q \models\left\langle\langle G\rangle \bigcirc \varphi \text { iff } \exists \sigma_{G} \in \Sigma_{G},\right. \text { such that } \\
& \forall \lambda \in \operatorname{comp}\left(q, \sigma_{G}\right), \text { we have } S, \lambda[1] \models \varphi ;
\end{aligned}
$$

$S, q \models\left\langle\langle G\rangle \square \square\right.$ iff $\exists \sigma_{G} \in \Sigma_{G}$, such that $\forall \lambda \in \operatorname{comp}\left(q, \sigma_{G}\right)$, we have $S, \lambda[u] \models \varphi$ for all $u \in \mathbb{N}$;

$S, q \models\langle\langle G\rangle\rangle \varphi \mathcal{U} \psi$ iff $\exists \sigma_{G} \in \Sigma_{G}$, such that $\forall \lambda \in \operatorname{comp}\left(q, \sigma_{G}\right)$, there exists some $u \in \mathbb{N}$ such that $S, \lambda[u] \models \psi$, and for all $0 \leq v<u$, we have that $S, \lambda[v] \models \varphi$.

$S, q \models K_{i} \varphi$ iff for all $q^{\prime}$ such that $q \sim_{i} q^{\prime}: S, q^{\prime} \models \varphi$;

$S, q \models E_{G} \varphi$ iff for all $q^{\prime}$ such that $q \sim_{G}^{E} q^{\prime}: S, q^{\prime}=\varphi$;

$S, q \models C_{G} \varphi$ iff for all $q^{\prime}$ such that $q \sim_{G}^{C} q^{\prime}: S, q^{\prime} \models \varphi$.

The other connectives ( $\wedge$ ", “ $\rightarrow$ ", “↔”) are assumed to be defined as abbreviations in terms of $\neg, \vee$. Also, $M_{i} \varphi$ means $\neg K_{i} \neg \varphi$, while $\langle\langle G\rangle\rangle \nabla \varphi$ is shorthand for $\neg\langle\langle G\rangle\rangle \square \neg \varphi$. We write $\langle\langle i\rangle\rangle$ rather than $\langle\langle\{i\}\rangle\rangle$. The cooperation modality $\langle\langle\rangle\rangle$ asserts that its argument is true on all computations, and thus acts like CTL's universal path quantifier "A". Similarly, $\langle\langle A g\rangle$ asserts that its argument is satisfied on at least one computation, and thus acts like the CTL path quantifier "E".

We now briefly mention two properties of ATEL. For any

$$
S=\left\langle Q, q_{0}, A g, A c_{1}, \ldots, A c_{n}, \sim_{1}, \ldots, \sim_{n}, \rho, \tau, \Phi, \pi\right\rangle,
$$

let the relation $R_{A g}$ between states in $Q$ be defined as:

$$
q_{1} R_{A g} q_{2} \text { iff } \exists j \in J_{A g}: \tau\left(q_{1}, j\right)=q_{2} .
$$

In other words, $q_{1} R_{A g} q_{2}$ holds if the grand coalition can enforce a transition from $q_{1}$ to $q_{2}$. Let

$$
S=\left\langle Q, q_{0}, A g, A c_{1}, \ldots, A c_{n}, \sim_{1}, \ldots, \sim_{n}, \rho, \tau, \Phi, \pi\right\rangle
$$

and

$$
S^{\prime}=\left\langle Q^{\prime}, q_{0}^{\prime}, A g, A c_{1}^{\prime}, \ldots, A c_{n}^{\prime}, \sim_{1}^{\prime}, \ldots, \sim_{n}^{\prime}, \rho^{\prime}, \tau^{\prime}, \Phi, \pi^{\prime}\right\rangle
$$

be two AAETSs. We say that $S^{\prime}$ is a subsystem of $S$, (notation $\left.S^{\prime} \sqsubseteq S\right)$ if there exists a relation

$$
\Re \subseteq Q \times Q^{\prime}
$$

such that:

1. $q_{0} \Re q_{0}^{\prime}$

2. $\forall q \in Q, q^{\prime} \in Q^{\prime}: q \Re q^{\prime} \Rightarrow\left(\pi(q)=\pi\left(q^{\prime}\right)\right)$

3. $\forall q_{1} \in Q, q_{1}^{\prime}, q_{2}^{\prime} \in Q^{\prime}$ :

$\left(\left(q_{1} \Re q_{1}^{\prime} \& q_{1}^{\prime} R_{A g}^{\prime} q_{2}^{\prime}\right) \Rightarrow\left(\exists q_{2} \in Q: q_{1} R_{A g} q_{2} \& q_{2} \Re q_{2}^{\prime}\right)\right)$

The relation $\Re$ is essentially half of a bisimulation between two Kripke models [3, p.64]: the final clause represents the "backward clause" for such relations, with the "forward clause" not being present in the conditions on $\Re$. We are interested in which formulas are preserved when going from $S$ to $S^{\prime}$, where $S^{\prime} \sqsubseteq S$. To this end, we define a universal and an existential sublanguage of ATEL, denoted $\mathcal{L}^{u}$ and $\mathcal{L}^{e}$, respectively. These languages are defined by the following grammars $(p \in \Phi)$ :

$$
\begin{aligned}
\mathcal{L}^{u} \quad v::= & p|\neg p| v \wedge v|v \vee v| K_{i} v\left|E_{G} v\right| C_{G} v \mid \\
& \langle\langle\rangle\rangle \bigcirc v|\langle\langle\rangle\rangle v|\langle\langle\rangle\rangle \square v \mid\langle\langle\rangle\rangle \mathcal{U} v \\
\mathcal{L}^{e} \quad \epsilon::= & p|\neg p| \epsilon \wedge \epsilon|\epsilon \vee \epsilon| M_{i} \epsilon|\langle\langle A g\rangle\rangle \in| \\
& \langle\langle A g\rangle\rangle \diamond \epsilon \mid\langle\langle A g\rangle \square \epsilon|\langle\langle A g\rangle\rangle \in \mathcal{U} \epsilon
\end{aligned}
$$

We now get the following result, which basically says that existential formulas are preserved when moving to a larger structure, whereas universal are preserved when restricting the structure.

Lemma 1. Let $S^{\prime} \sqsubseteq S, v \in \mathcal{L}^{u}, \epsilon \in \mathcal{L}^{e}$. Then:

$$
S^{\prime}, q \models \epsilon \Rightarrow S, q \models \epsilon \text { and } S, q \models v \Rightarrow S^{\prime}, q \models v
$$

\section{KNOWLEDGE AND SOCIAL LAWS}

We now present our formal framework of social laws and demonstrate how knowledge is incorporated into the framework by investigating various knowledge properties. As in [16], a social law consists of two parts: 
1. An objective, which represents what the society aims to achieve by the introduction, or adoption of the law. This objective can now refer to knowledge. In human societies, for example, the objective of a law might be to ensure that bank card PIN numbers remain private to the card holder.

2. A behavioural constraint, which corresponds to the requirements that a law places on the members of a society. A behavioural constraint corresponding to the objective of ensuring PIN numbers remain private might be to forbid the action of reading another person's PIN number.

Now we represent an objective for a social law as a formula of ATEL, with the same intuition that a social law is effective if it ensures that the objective is satisfied. ATEL inherits all the properties of ATL, allowing us to reason about what certain coalitions can bring about, as well as the ability to express liveness and safety properties from CTL, but now we can also express knowledge properties in order to reason about what agents and coalitions of agents should and shouldn't know.

As in previous work, we model a behavioural constraint, $\beta$, as a function $\beta: A c_{A g} \rightarrow 2^{Q}$, with the intended interpretation that if $q \in \beta(\alpha)$, then action $\alpha$ may not be performed when the system is in state $q$ - that is, $\alpha$ is "forbidden" in state $q$. We require that any behavioural constraint is "reasonable", in that it always permits an agent to have at least one action left that can be performed in any state:

$$
\forall i \in A g, \forall q \in Q, \exists \alpha \in \operatorname{options}(i, q) \text { s.t. } q \notin \beta(\alpha) .
$$

We can now see what it means for a behavioural constraint $\beta$ to be implemented in an AAETS $S$ : it means eliminating from $S$ all transitions that are forbidden by $\beta$. The operation of implementing a behavioural constraint is thus an update on AAETSs, in the sense that it results in a new AAETS, which potentially satisfies different formulae. We denote the AAETS obtained from $S$ by implementing $\beta$ by $S \dagger \beta$. Formally, if

$$
S=\left\langle Q, q_{0}, A g, A c_{1}, \ldots, A c_{n}, \sim_{1}, \ldots, \sim_{n}, \rho, \tau, \Phi, \pi\right\rangle
$$

is an AAETS, and $\beta$ is a behavioural constraint on $S$, then $S \dagger \beta=$

$$
\left\langle Q, q_{0}, A g, A c_{1}, \ldots, A c_{n}, \sim_{1}, \ldots, \sim_{n}, \rho^{\prime}, \tau^{\prime}, \Phi, \pi\right\rangle,
$$

where:

1. $\forall \alpha \in A c: \quad \rho^{\prime}(\alpha)=\rho(\alpha) \backslash \beta(\alpha)$

2. $\forall q \in Q, \forall j \in J_{A g}: \quad \tau^{\prime}(q, j)=$

$$
\begin{cases}\tau(q, j) & \text { if }(q, j) \in \operatorname{dom} \tau \text { and } \forall i \in A g, q \notin \beta\left(j_{i}\right) \\ \text { undefined } & \text { otherwise }\end{cases}
$$

3. All other components of $S \dagger \beta$ are as in $S$.

A social law over AAETS $S$ is then a pair $(\varphi, \beta)$ where:

- $\varphi$ is an ATEL formula called the objective of the law;

- $\beta: A c_{A g} \rightarrow 2^{Q}$ is a behavioural constraint on $S$.

The feasibility problem for social laws as defined in [16] is as follows:

Given an AAETS $S$ and a formula $\varphi$, intended to represent an objective, does there exist a behavioural constraint $\beta$ that is effective for $\varphi$ in $S$, i.e., such that $S \dagger \beta \models\langle\langle\rangle\rangle \square \varphi$.
It was proved in [16] that, for objectives expressed in ATL, the feasibility problem is NP-complete. We now show that, for ATEL objectives, the problem is no worse.

THEOREM 1. The feasibility problem for objectives expressed as arbitrary ATEL formulae is NP-complete.

Proof. Since feasibility for ATEL objectives subsumes feasibility for ATL objectives, we only need to prove the upper bound. This follows from the fact that we can guess a behavioural constraint $\beta$, which will be polynomial in the size of $S=\left\langle Q, q_{0}, A g, A c_{1}, \ldots, A c_{n}, \sim_{1}, \ldots, \sim_{n}, \rho, \tau, \Phi, \pi\right\rangle$, and then verify that $S \dagger \beta \models\langle\langle\rangle\rangle \square \varphi$. The process of generating $S \dagger \beta$ can easily be done in time polynomial in the size of $S$ (we simply delete from $S$ all transitions forbidden by $\beta$ ), and since model checking for ATEL is in P, the result follows.

\subsection{Knowledge Properties}

There are certain knowledge properties that are of interest to us. A knowledge pre-condition is the information that an agent must have in order to be able to successfully carry out an action or plan. Knowledge pre-condition formulae take the form $K_{i} \varphi \rightarrow \psi$. This formula states that agent $i$ knowing the fact $\varphi$ implies that the fact (state of affairs) $\psi$ should hold. These knowledge pre-condition formulae can be used as ATEL objectives in our social laws. Let us define $\llbracket i \rrbracket \varphi$ as the dual of $\langle\langle i\rangle\rangle \varphi$, so that $\llbracket i \rrbracket \varphi$ means that $i$ cannot prevent the others to achieve $\varphi$ (whichever strategy $i$ choses, the others can complement it in such a way that $\varphi$ ).

Then, a property like

$$
K_{i} \varphi \rightarrow \llbracket i \rrbracket(\bigcirc \psi \vee \bigcirc \bigcirc \psi \vee \bigcirc \bigcirc \bigcirc \psi)
$$

expresses that, if the gate-controller $(i)$ on a crossing knows that the train is waiting to cross $(\varphi)$, then, whatever the gates do, the train should be able to safely cross $(\psi)$ in the 'near future' (i.e., within the next three time steps). We can extend this example immediately to cases where $\psi$ is itself epistemic: if the train $(j)$ knows that the constraint (1) applies, and he wants to safely cross, he should notify the the gate-controller of this:

$$
K_{j} \varphi \rightarrow\langle\langle j\rangle\rangle \bigcirc K_{i} \varphi
$$

Implied knowledge is knowledge that comes about as a result of the performance of actions, which lead to a change in the current state of affairs: they present themselves as $\varphi \rightarrow K_{i} \psi$. This formula states that the state of affairs $\varphi$ should bring about the knowledge of the fact $\psi$ to agent $i$.

This type of formulae can also be used in the objectives to our social laws. For example, the following could express that everytime that train $i$ is able to pass a crossing, the gate at that crossing should know it.

$$
\left\langle\langle i\rangle \bigcirc \psi \rightarrow K_{j} \varphi\right.
$$

Finally, nested knowledge is information that the agents have about what other agents know. For instance, $\llbracket i \rrbracket \triangleright \psi \rightarrow$ $K_{i} E_{G} \varphi$ expresses that if $i$ cannot ensure that $\psi$ will ever become true, he should make sure (know) that everybody in $G$ knows this. Nested knowledge can also play the role of a pre-condition (I should know that you know we have a meeting at the station, before me going there, for instance). 


\section{A CASE STUDY}

In this scenario, there is a train on a circular track, which at one point crosses a road (see Figure 1). The place where the track crosses the road (level crossing) is controlled by gates operated by a gate controller agent. If the train moves on to the level crossing while the gates are closed, the train will be in a crash situation, in which it has crashed into a car on the crossing. Also, if the gates close while the train is on the crossing, a crash will occur. We are interested in social laws that can prevent such situations from arising.

This system consists of two agents called $t$ and $g$, where $t$ is the agent representing the train and $g$ is the gate controller agent. The train can be in one of four states, "staA" (train is at station A, which is the initial state of the train), "staB" (train stopped at station B), "wait" (waiting to use the crossing) and "oncr" (the train is on the crossing). The gates can only be in two states, either "open" (gates are open to the train) or "closed" (gates are closed to the train).

There is a communication medium by which the train and gate controller agent can exchange messages about their local states. The communication works by signals sent and received via an aerial on each of the agents. Signals received from the gates controller agent are stored in a local variable of the train called Flag. Similarly, signals received from the train are stored in a local variable of the gate controller agent called Flagt.

The train has six actions available to it: move $_{t}$, moveann $_{t}$, tell-away tell-wait $_{t}$, tell-oncr ${ }_{t}$, and $i_{\text {ille }}$. The $i d l e_{t}$ action is the identity which causes no change in the train's state. If the train executes a move $_{t}$ action while it is at staA, then it goes to $s t a B_{t}$; executing move $_{t}$ while at $s t a B_{t}$ causes a

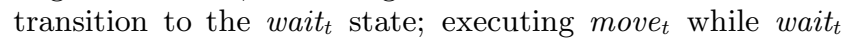
causes a transition to the oncr $_{t}$ state; and, finally, executing move $_{t}$ while oncr $_{t}$ causes a transition to $s t a A_{t}$, as long as the gates were not in the closed $_{g}$ state at the same time as the

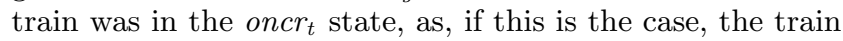
is said to have crashed and is forced to idle $e_{t}$ indefinitely.

The moveann $n_{t}$ action causes the same state transitions as the movet action, but additionally the train sends its new location information to $g$. The actions prefixed with tell have the effect of telling the gates that the location of

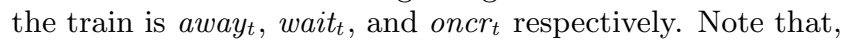
without any social law in place, it is not guaranteed that the train tells his location truthfully (e.g. performing tell-wait when actually in state $o n c r_{t}$ )!

The gates controller has five actions available to it: gates $_{g}$,

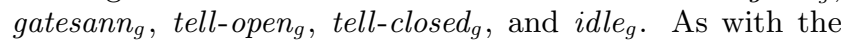
train, this idle $_{g}$ action causes no change in the state of the gates. The gatesg action causes the position of the gates to be toggled, i.e., performing gates $_{g}$ when the gates are closed $_{g}$ will result in the gates being openg and vice versa. The gatesann $_{g}$ action causes the same transitions as the gates $_{g}$ action, but the gate controller agent sends its new status to the train agent. The actions prefixed with tell have the effect of telling the train that the new status of the gates is closed $_{g}$ and open $_{g}$ respectively.

A state in our system is defined to be a tuple

$$
\left\langle\text { Loc }_{t}, \text { Flag }_{g}, \text { Status }_{g}, \text { Flag }_{t}\right\rangle
$$

where:

$$
\text { - } \operatorname{Loc}_{t} \in\left\{s t a A_{t}, s_{t a B_{t}}, \text { wait }_{t}, \text { oncr }_{t}\right\}
$$

- Flag $_{g} \in\{$ true, false $\}$

- Status $_{g} \in\left\{\right.$ open $_{g}$, closed $\left._{g}\right\}$

- Flag $_{t} \in\{a, w, o\}$

We assume that we have atoms in the object language (like Flag $_{t}=w$ ) corresponding to these states. We will refer to this tuple generally as $\langle W, X, Y, Z\rangle$. We let $q_{i}$ denote the $i$ th component of $q$. Now, $\left\langle q_{1}, q_{2}, q_{4}\right\rangle$ is the local state of agent $t$ and $\left\langle q_{2}, q_{3}, q_{4}\right\rangle$ is the local state of agent $g$. The initial state (or root) of our system is $\rho=$ $\left\langle\right.$ staA $_{t}$, false $\left._{\text {, closed }}, a\right\rangle$.

Given two states $q, r \in Q$, then the epistemic accessibility relation for agent $t$ is given as: $q \sim_{t} r$, iff $q_{1}=r_{1}$, $q_{2}=r_{2}$ and $q_{4}=r_{4}$. The epistemic accessibility relation for agent $g$ is given as: $q \sim_{g} r$, iff $q_{2}=r_{2}, q_{3}=r_{3}$ and $q_{4}=r_{4}$. Let us call this overall system $S$. The transitions are given in Figure 5, where the first line for instance represents that a move in any state $\left\langle\right.$ sta $\left.A_{t}, X, Y, Z\right\rangle$, when done jointly with any action $a c_{g}$ of the gates, results in any of $\left\langle s t a B_{t}, X^{\prime}, Y^{\prime}, Z\right\rangle$ states, where the precise $X^{\prime}$ and $Y^{\prime}$ values (denoting the state of the gates), depend on $X, Y$, and $a c_{g}$, the location of the train changes from station $A$ to station $B$, and the value of $F$ lag $_{t}$, stored in the variable $Z$, remains the same.

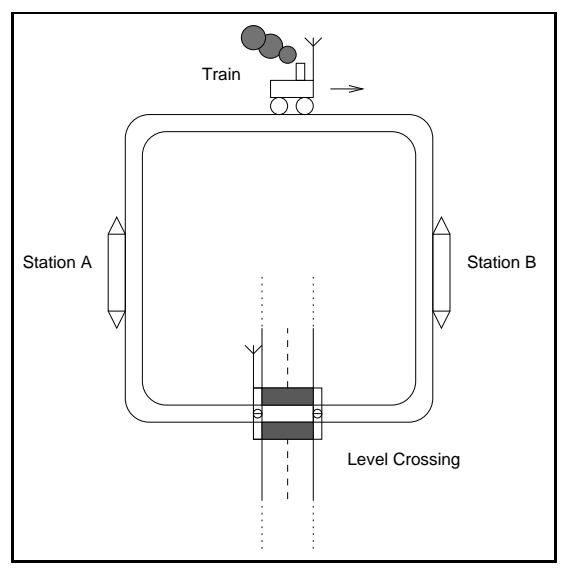

Figure 1: The Level Crossing system.

\subsection{Epistemic Social Laws}

Now we can formulate some objectives for social laws. They have the general format $K_{i} \varphi \rightarrow \psi$, where $\psi$ is some property under control of agent $i$. This format makes perfect sense: (only) if an agent knows certain preconditions, can we require from him to take appropriate action. Let us hence say that such an objective is feasible for agent $i$.

$$
O_{1}=K_{t} \text { wait }_{t} \rightarrow\left(\text { Flag }_{t}=w\right)
$$

The objective $O_{1}$ is equivalent to $O_{1}^{\prime}=$ wait $_{t} \rightarrow\left(\right.$ Flag $_{t}=$ $w$ ) (if this is not immediately clear, it should become clear once we have discussed the notion of a local proposition). Property (4) denotes that the train is accurate with respect to recording his waiting state. We give a 'weakest' constraint $\beta_{1}$ that implements this social law, which can be verified by checking $S \dagger \beta_{1}, r \models\langle\langle\rangle\rangle \square O_{1}^{\prime}$. The constraint $\beta_{1}$ works 


\begin{tabular}{|c|c|c|}
\hline Current State & Actions & Resulting States \\
\hline 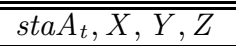 & move $_{t}, a c_{g}$ & $\overline{s t a B_{t}, X^{\prime}, Y^{\prime}, Z}$ \\
\hline$s t a B_{t}, X, Y, Z$ & move $_{t}, a c_{g}$ & wait $_{t}, X^{\prime}, Y^{\prime}, Z$ \\
\hline wait $_{t}, X, Y, Z$ & move $_{t}, a c_{g}$ & oncr $_{t}, X^{\prime}, Y^{\prime}, Z$ \\
\hline oncr $_{t}, X, Y, Z$ & move $_{t}, a c_{g}$ & $\operatorname{sta}_{t}, X^{\prime}, Y^{\prime}, Z$ \\
\hline$W, X$, closed $_{g}, Z$ & $a_{t}$, gates $_{g}$ & $W^{\prime}, X$, open $_{g}, Z^{\prime}$ \\
\hline$W, X$, open $_{g}, Z$ & $a c_{t}$, gates $_{g}$ & $W^{\prime}, X, \operatorname{closed}_{g}, Z^{\prime}$ \\
\hline$W, X, Y, Z$ & $i d l e_{t}, a c_{g}$ & $W, X^{\prime}, Y^{\prime}, Z$ \\
\hline$W, X, Y, Z$ & $a c_{t}, i d l e_{g}$ & $W^{\prime}, X, Y, Z^{\prime}$ \\
\hline $\operatorname{staA}_{t}, X, Y, Z$ & moveann $_{t}, a c_{g}$ & $\operatorname{sta} B_{t}, X^{\prime}, Y^{\prime}, a$ \\
\hline $\operatorname{staB}_{t}, X, Y, Z$ & moveann $_{t}, a c_{g}$ & wait $_{t}, X^{\prime}, Y^{\prime}, w$ \\
\hline wait $_{t}, X, Y, Z$ & moveann $_{t}, a c_{g}$ & oncr $_{t}, X^{\prime}, Y^{\prime}, o$ \\
\hline oncr $_{t}, X, Y, Z$ & moveann $_{t}, a c_{g}$ & $\operatorname{sta}_{t}, X^{\prime}, Y^{\prime}, a$ \\
\hline$W, X$, closed $_{g}, Z$ & $a_{t}$, gatesann $n_{g}$ & $W^{\prime}$, true open $_{g}, Z^{\prime}$ \\
\hline$W, X$, open $_{g}, Z$ & $a_{t}$, gatesann & $W^{\prime}$, false, closed $_{g}, Z^{\prime}$ \\
\hline$W, X, Y, Z$ & tell-away ${ }_{t}, a c_{g}$ & $W, X^{\prime}, Y^{\prime}, a$ \\
\hline$W, X, Y, Z$ & tell-wait $_{t}, a c_{g}$ & $W, X^{\prime}, Y^{\prime}, w$ \\
\hline$W, X, Y, Z$ & tell-oncr,$a c_{g}$ & $W, X^{\prime}, Y^{\prime}, o$ \\
\hline$W, X, Y, Z$ & $a c_{t}$, tell-closed ${ }_{g}$ & $W^{\prime}$, false, $Y, Z^{\prime}$ \\
\hline$\overline{W, X, Y, Z}$ & $a c_{t}$, tell-open ${ }_{g}$ & $W^{\prime}$, true $, Y, Z^{\prime}$ \\
\hline
\end{tabular}

Figure 2: State Transitions

as follows: in $\left\langle s t a B_{t}, X, Y, Z\right\rangle$, if $Z \neq w$, the action for $t$ forbidden by $\beta_{1}$ is move (since this would inaccurately leave the Flag $_{t}$ to $a$ or $o$ ); moreover, in any state $\left\langle\right.$ wait $\left._{t}, X, Y, Z\right\rangle$, constraint $\beta_{1}$ forbids $t$ to perform any tell action, except when it is tell-wait. Loosely speaking: the train is accurate about his waiting, if he announces it when he starts to wait, and, once waiting, never tells anything otherwise. We claim that moreover $\beta_{1}$ is a weakest constraint for its aims: any constraint $\beta$ that forbids less than $\beta_{1}$ has the property that $S \dagger \beta, r \models\langle\langle\{t, g\}\rangle\rangle\rangle \neg O_{1}$.

The converse of (4) would mean that the train is truthful with respect to the waiting state:

$$
\mathrm{O}_{2}=K_{t}\left(\text { Flag }_{t}=w\right) \rightarrow \text { wait }_{t}
$$

Again, this property is equal to $O_{2}^{\prime}=\left(\right.$ Flag $\left._{t}=w\right) \rightarrow$ wait. . The behavioural constraint $\beta_{2}$ that makes this social law effective is to forbid $t$ to falsely suggest that it is waiting: $t$ is only allowed to perform tell-wait $t_{t}$ in any $\left\langle\right.$ wait $\left._{t}, X, Y, Z\right\rangle$, moreover, $\beta_{2}$ forbids $t$ to perform a move $t_{t}$ in $\left\langle\right.$ wait $\left._{t}, Y, Z, w\right\rangle$, since otherwise it would falsely suggest that it is still waiting (of course, the train can still leave this state truthfully, by performing a moveannt-action). The fact that indeed $\beta_{2}$ implements $O_{2}^{\prime}$ is verified by showing $S \dagger \beta_{2}, \rho \models\langle\langle\rangle\rangle \square O_{2}^{\prime}$. As an aside, note that the constraint $\beta_{4}$ that never allows the train to do a move $t$ action and only allows $t$ to perform tell-wait when wait $t_{t}$ is true, is a way to implement the objective $O_{4}=\left(\left(\right.\right.$ Flag $\left._{t}=w\right) \rightarrow$ wait $\left._{t}\right)$.

Now we observe the following: $S \dagger \beta_{1} \dagger \beta_{2}, r \models$ wait $_{t} \rightarrow$ $K_{g}$ wait $_{\text {. }}$ This is interesting, since to make the gates know that the train is waiting, has become a train-feasible objective in $S \dagger \beta_{1} \dagger \beta_{2}$ : he just has to accurately and truthfully set Flagt to $w$ !

Now consider the following objective:

$$
O=\left\langle\langle\rangle \square \square \neg\left(\text { oncr }_{t} \wedge \text { closed }_{g}\right) \wedge\langle\langle g, t\rangle\rangle\right\rangle \text { onct }_{t}
$$

This objective combines a safety property (the train is never on the crossing while it is closed) with a liveness prop- erty (the train will eventually pass the crossing). The question is whether we can 'break' this objective 'down' into a number of constraints that are feasible for $t$ or $g$. Here is a high level description: Let $O^{\prime}$ be the conjunction of:

$$
\begin{array}{ll}
\left(K_{t} \text { wait }_{t} \rightarrow K_{g} \text { wait }_{t}\right) & \left(K_{g} \text { wait }_{t} \rightarrow \llbracket g \rrbracket \triangleright \text { open }_{g}\right) \\
\left(K_{g} \text { open }_{g} \rightarrow K_{t} \text { open }_{g}\right) & \left(K_{t} \text { open }_{g} \rightarrow\langle\langle t\rangle\rangle \text { oncr }_{t}\right) \\
\left(K_{g} \text { closed }_{g} \rightarrow K_{t} \text { closed }_{g}\right) & \left(K_{t} \text { closed }_{g} \rightarrow \llbracket t \rrbracket \square \neg \text { oncr }_{t}\right)
\end{array}
$$

Objective $O^{\prime}$ states that $t$ should inform $g$ about waiting, and $g$ should inform $t$ about the states of the gates. Recall that $\llbracket i \rrbracket \nabla \varphi$ means that the other agents can ensure $\nabla \varphi$, and, if $\varphi$ is 'under control' of $i$, then $\llbracket i \rrbracket \diamond \varphi$ means that $i$ cannot avoid that $\varphi$ will eventually be true. Keeping this in mind, $O^{\prime}$ then also requires that if $g$ knows that $t$ is waiting, it cannot but avoid that eventually the gates will be open; if $t$ knows the gates to be open, it will eventually pass the crossing, and, finally, if $t$ knows the gates are closed, it will never attempt to cross. We claim that $O^{\prime}$ can be indeed turned into a set of feasible laws for $g$ and $t$, and also that the implemented law for $O^{\prime}$ guarantees $O$.

We demonstrated how to make the first implication feasible for $t$ by imposing suitable constraints; the same can be done for the other implications. We will now demonstrate how to actually model-check such knowledge properties.

\subsection{Model Checking Some Properties}

There are certain knowledge properties that need to be satisfied in the level crossing example. We obtain them using social laws. In order to test knowledge properties, we use a model checker called MOCHA, which takes as input an ATS described using a high-level language called REACTIVE MODULES. We programmed the system in this language and also have modified versions of the system, which incorporate social laws. It is important to note that we are not adding to the model checking theory, simply making use of it.

The first knowledge property that we wish to investigate is that the gates always know when the train is waiting. More formally, we want to verify that, given a state $q$ in which $q \models$ wait $_{t}$, we also have $q \models K_{g}$ wait $_{t}$. The intuition behind this knowledge property is that if the train is waiting at the gates for them to be opened, this can only happen if the gate controller agent knows that the train is waiting.

Now we wish to verify properties involving knowledge by using a standard model checker that does not deal with epistemic operators, in our case MOCHA ([1]). To do this, we employ the machinery of local propositions, as introduced in [4] and applied to model checking epistemic properties in the context of linear temporal logic in [17]. We give an informal explanation; for details the reader should consult [17].

A proposition $\varphi$ is $i$-local in a system $S$ if

$$
\forall q, q^{\prime} \in S:\left(\left(q \sim_{i} q^{\prime}\right) \Rightarrow\left(S, q \models \varphi \Leftrightarrow S, q^{\prime} \models \varphi\right)\right)
$$

that is, an $i$-local proposition never changes truth value within an $i$-equivalence class of states. This formalises the idea that such propositions depend on what the agent can observe. We immediately see that in the train and gates example, every proposition about the location of the train and the flag of the gates is $t$-local, likewise are assertions about the status of the gates and the flag of the train local for the gates. Now, generalizing the linear temporal logic analysis from [17] to the branching time context of this paper, we have the following: 
Theorem 2. Suppose that $\varphi$ is i-local. Then $S, q \models \varphi$ and $S, q_{0} \models\langle\langle\rangle\rangle \square(\varphi \rightarrow \psi)$ are sufficient to prove both $S, q \models$ $K_{i} \psi$ and $S=\varphi \rightarrow K_{i} \psi$. In such a case, $\varphi$ is called $i$-local for $\psi$.

This theorem immediately shows that the laws $\mathrm{O}_{2}$ and $\mathrm{O}_{2}^{\prime}$ are equivalent. To show that $q \models K_{t}$ wait $_{t}$ in any waiting state $q$, we must first find a $g$-local proposition for wait . $_{\text {. }}$ We can take $\left(\right.$ Flag $\left._{t}=w\right)$ : To show that $\left(\right.$ Flag $\left._{t}=w\right)$ is $g$-local in $S \dagger \beta_{2}$, we need to verify that $\forall q, r\left(\left(q \sim_{g} r\right) \Rightarrow\right.$ $\left(q \models\right.$ Flag $_{t}=w \Leftrightarrow r \models F$ lag $\left.\left._{t}=w\right)\right)$, which is obvious, since Flag $g_{t}$ is part of $g$ 's local state. Now, to apply Theorem 2, we must show that $S \dagger \beta_{2}, r \models\langle\langle\rangle\rangle\left(\right.$ Flag $\left._{t}=w\right) \rightarrow$ wait $_{t}$ holds. We do this by model checking the following мOCHA ATL specification in $S \dagger \beta_{2}$ :

$$
\text { «८> G }((\text { tFlag }=\mathrm{w}) \Rightarrow(\text { tState }=\text { wait }))
$$

Since the answer to this is positive, we have verified the desired property in $S \dagger \beta_{2}$. Note that this is relative to a state $q$. We now show how we can check this property across all states of the system. The only thing we required in $q$ is that $q \models$ wait $_{t}$. So now we can check the following property across all states, in $S \dagger \beta_{2}$ :

$$
\text { wait }_{t} \rightarrow K_{g} \text { wait }_{t}
$$

Now we model check the following:

$$
\text { « }
$$

When this formula is model checked in the original system $S$ it fails: This is as expected, as there is nothing in-built in $S$ to guarantee that the train is truthful. However, model checking the above formula in $S \dagger \beta_{2}$ passes, which shows that this social law is effective.

Now we look at a nested knowledge formula:

$$
\text { wait }_{t} \rightarrow K_{t} K_{g} \text { wait }_{t}
$$

We briefly sketch how to do this in the system that is constrained with $\beta_{4}$. By Theorem 2 , it is sufficient to show that wait $t_{t}$ is $t$-local for $K_{g}$ wait $_{t}$. That is, wait is $t$-local (which is obviously the case) and $S \dagger \beta_{4}, \rho \models\langle\langle\rangle\rangle \square\left(\right.$ wait $_{t} \rightarrow$ $K_{g}$ wait $\left._{t}\right)$. This can be either model checked or established from (6), the fact that $S \dagger \beta_{4} \sqsubseteq S \dagger \beta_{2}$ and Lemma 1. We can now apply Theorem 2 (take $\varphi=$ wait $_{t}$ and $\psi=K_{g}$ wait $_{t}$ ), to conclude that $S \dagger \beta_{4} \models$ wait $_{t} \rightarrow K_{t} K_{\text {g }}$ wait $_{t}$.

The social laws imposed on our system are quite restrictive in that agent $t$ knows everything about the state of agent $g$, and agent $g$ knows a lot about the state of agent $t$. The knowledge that each of the agents have is necessary to ensure the system runs efficiently and that no crash situations occur. However, when the train is at $s t a A_{t}$ or $s t a B_{t}$, it is not beneficial to the system that agent $g$ should know which of the two states the train is at, only that the train is away, in order to close the gates. We show this by investigating the following property in $S$ :

$$
\operatorname{sta} A_{t} \rightarrow M_{g} \neg \operatorname{sta} A_{t}
$$

This property states that if the train is at station A, agent $g$ considers it possible that in fact, the train is not at station A. To check whether (8) holds across all states of our system $S$, we model check the following formula:

$$
\bigwedge_{x, y}\left(\left\langle\langle\rangle \square \square\left(s t a A_{t} \wedge x \wedge y\right) \rightarrow\langle\langle\rangle\rangle \square\left(\neg s t a A_{t} \wedge x \wedge y\right)\right)\right.
$$

where the conjunction is taken over all

$$
x \in\left\{\text { open }_{g}, \text { closed }_{g}\right\}
$$

and

$$
y \in\left\{\text { Flag }_{t}=a, \text { Flag }_{t}=w, \text { Flag }_{t}=o\right\} .
$$

This requires performing six different model checking problems, for each of the different values of $x$ and $y$. After performing this model checking, as the formula passed each time, we have shown that (8) holds. Finally notice that even if the constraints concerning accuracy and truthfulness of both agents are implemented, (8) holds, since the most specific information that $t$ will give when being at station $A$ or $B$ will be that he is 'away'.

\section{RELATED WORK}

As our framework is an extension of [16], it is clearly related to this work. Our approach is also related to the original frameworks of Moses and Tennenholtz, and that of Shoham and Tennenholtz. Shoham and Tennenholtz were the first to articulate the notion of social laws for multiagent systems, and set up a basic formal framework within which computational questions about social laws could be formulated ([14]).

In [8], Moses and Tennenholtz developed an deontic epistemic logic for representing properties of multiagent systems with normative structures in place. This framework is semantically similar to ours, not least because they also define epistemic accessibility relations, intended to interpret the agents' knowledge. These relations are defined to be equivalence relations representing states that are indistinguishable to agent $i$. We capture knowledge in precisely this way. However, Moses and Tennenholtz do not go on to make use of these relations in formulating social laws containing knowledge, as we do in this paper.

Another body of work that can be deemed similar to ours, is that of Rohit Parikh et al. In [9], they put forward the notion of knowledge based obligation. Here, the idea is that obligations are dependent on what the agent knows. For example, if a doctor does not know that a patient is ill, there is no obligation for the doctor to treat the patient. However, if the doctor does know that the patient is ill, this creates an obligation for the doctor to treat the patient. Obligations can also be over-ridden by more relevant information. Obligations are intended to further some general good and given the choice, agents should do what is best for society. These obligations can be thought of as similar to our social laws, where, for example, if the train moves from the crossing to station A, it is obliged to inform the gates of its next state.

\section{CONCLUSION}

In this paper we have shown how the social laws framework of van der Hoek et al [16] can naturally be extended to incorporate the notion of knowledge. In order to express such social laws we use the language of ATEL, essentially ATL with epistemic extensions. We demonstrated the power of such a framework with the use of a case study, in which many interesting knowledge properties were investigated. In particular, we showed how overall objectives of the system can be broken down into feasible properties for the agents, 
involving laws for each agent $i$ of type $K_{i} \varphi \rightarrow \psi$, where $\psi$ is under control of $i$, possibly after implementing a social law.

We also demonstrated how several different types of knowledge, including knowledge pre-conditions, implied knowledge, and nested knowledge can be verified using an ATL model checker (MOCHA). We showed how ATEL formulae can be reduced to ATL formulae with the use of local propositions substituted for knowledge. This allowed us to verify such properties with a standard model checker that does not deal with epistemic operators.

Now we have extended the framework further, there are still many avenues for further investigation. It is interesting to incorporate the notions of minimality and simplicity ([6]) into the framework, and investigate them in this setting. The idea behind minimality and simplicity is essentially not to 'over-constrain' the system when achieving its objective. Second, the social laws are not yet expressible in the object language: we state them in ATEL, but then deal with them only semantically.

We are currently extending the framework to allow explicitly in the object language to not only refer to obligations and duties, but also to specific actions or strategies, giving an expressive language to reason about who should achieve what in which way. One attempt to add terms of actions in an ATL-like framework is to be found in [15], be it in a setting that does not address knowledge.

Finally, we like to have a general theory that predicts when an overall objective can be translated in individual feasible objectives, a question that is obviously related to the choice of how to partition the states in local substates. We think the analysis of [19] maybe helpful here. In [19], a notion of co-operation and control is studied in which each agent has the power to 'set' or 'unset' certain propositional atoms. This may be relevant for our social laws in the following sense: if $\varphi$ is the objective of a social law, and all the atoms occurring in $\varphi$ are $p_{1}, \ldots p_{k}$, then the agents that control those atoms have a kind of 'responsibility' to implement the social laws that is greater than those who cannot influence the truth of $\varphi$ directly.

\section{REFERENCES}

[1] R. Alur, L. de Alfaro, T. A. Henzinger, S. C. Krishnan, F. Y. C. Mang, S. Qadeer, S. K. Rajamani, and S. Taşiran. MOCHA user manual. University of Berkeley Report, 2000.

[2] R. Alur, T. A. Henzinger, and O. Kupferman. Alternating-time temporal logic. Journal of the ACM, 49(5):672-713, Sept. 2002.

[3] P. Blackburn, M. de Rijke, and Y. Venema. Modal Logic. Cambridge University Press: Cambridge, England, 2001.

[4] K. Engelhardt, R. van der Meyden, and Y. Moses. Knowledge and the logic of local propositions. In Proceedings of the 1998 Conference on Theoretical Aspects of Reasoning about Knowledge (TARK98), pages 29-41, 1998.

[5] R. Fagin, J. Y. Halpern, Y. Moses, and M. Y. Vardi. Reasoning About Knowledge. The MIT Press: Cambridge, MA, 1995.

[6] D. Fitoussi and M. Tennenholtz. Choosing social laws for multi-agent systems: Minimality and simplicity. Artificial Intelligence, 119(1-2):61-101, 2000.
[7] J.-J. C. Meyer and W. van der Hoek. Epistemic Logic for AI and Computer Science. Cambridge University Press: Cambridge, England, 1995.

[8] Y. Moses and M. Tennenholtz. Artificial social systems. Computers and AI, 14(6):533-562, 1995.

[9] R. Parikh, E. Pacuit, and E. Cogan. The logic of knowledge based obligation. Presented at DALT 2004, available at ww. cs.gc. cuny.edu/ epacuit/docs/kbo.pdf.

[10] Y. Shoham and M. Tennenholtz. Emergent conventions in multi-agent systems. In C. Rich, W. Swartout, and B. Nebel, editors, Proceedings of Knowledge Representation and Reasoning (KRER-92), pages 225-231, 1992.

[11] Y. Shoham and M. Tennenholtz. On the synthesis of useful social laws for artificial agent societies. In Proceedings of the Tenth National Conference on Artificial Intelligence (AAAI-92), San Diego, CA, 1992.

[12] Y. Shoham and M. Tennenholtz. On social laws for artificial agent societies: Off-line design. Artificial Intelligence, 73(1-2):231-252, 1995.

[13] Y. Shoham and M. Tennenholtz. On social laws for artificial agent societies: Off-line design. In P. E. Agre and S. J. Rosenschein, editors, Computational Theories of Interaction and Agency, pages 597-618. The MIT Press: Cambridge, MA, 1996.

[14] Y. Shoham and M. Tennenholtz. On the emergence of social conventions: Modelling, analysis, and simulations. Artificial Intelligence, 94(1-2):139-166, July 1997.

[15] W. van der Hoek, W. Jamroga, and M. Wooldridge. A logic for strategic reasoning, 2005. See elsewhere in these proceedings of AAMASO5.

[16] W. van der Hoek, M. Roberts, and M. Wooldridge. Social laws in alternating time: Effectiveness, feasibility, and synthesis. Technical Report ULCS-04-017, The University of Liverpool, 2005. To appear in the journal Synthese.

[17] W. van der Hoek and M. Wooldridge. Model checking knowledge and time. In D. Bos̆nački and S. Leue, editors, Model Checking Software, Proceedings of SPIN 2002 (LNCS Volume 2318), pages 95-111. Springer, Germany, 2002.

[18] W. van der Hoek and M. Wooldridge. Time, knowledge, and cooperation: Alternating-time temporal epistemic logic and its applications. Studia Logica, 2003.

[19] W. van der Hoek and M. Wooldridge. On the logic of co-operation and propositional control. Artificial Intelligence, 64(1-2):81-119, 2005. 